\title{
Ups and downs of a peer-based smoking cessation intervention help tailored to hospital-employees with low socioeconomic status: The RESPEKT Study
}

\author{
Charlotta Pisinger ${ }^{1,2}$, Maj-Britt Bjerre Koch ${ }^{3}$, Else Hjortsø ${ }^{4}$, Torben Jørgensen ${ }^{2,5}$, Charlotte Glümer ${ }^{6}$
}

\begin{abstract}
INTRODUCTION Smoking is one of the most important determinants of socioeconomic inequality in mortality. Few studies have tested which interventions are effective in smokers with low socioeconomic status (SES).

MEthods All hospitals in the Capital Region of Denmark were included and randomized to intervention or control groups. The target-group was smokers with low SES. Intervention hospitals: smokers in the target-group assisted researchers to tailor a group-based smoking cessation intervention. Further they helped recruiting smoking colleagues and motivating them to stay abstinent. Control hospitals: 'as usual'. Unforeseen organizational challenges led to a change of study design; the hospital-level assessment was reduced to two cross-sectional surveys.

RESULTS Response rates in hospitals' smoking status survey were very low. Smoking status was reported by 1876 out of 7003 employees at baseline and 2280 out of 7496 employees at 1-year follow-up. Two cross-sectional surveys showed no significant difference in self-reported smoking at 1-year follow-up between intervention and control hospitals $(\mathrm{p}=0.262)$. We recruited 100 smokers in the group-based smoking cessation intervention tailored to smokers with low SES (corresponding to approx. $10 \%$ of smokers in target-group); $32.4 \%$ of these were validated as continuously abstinent at 6 months follow-up.

CONCLUSIONS Involving smokers with low SES as partners at an early stage of study design facilitated both recruitment and development of the intervention. Despite high validated long-term abstinence rates in smoking cessation groups in the intervention hospitals we found no apparent effect of the intervention at hospitallevel after one year. However, larger involvement of the target-group seems feasible and is recommended.
\end{abstract}

AFFILIATION

1 Center for Clinical Research and Prevention, BispebjergFrederiksberg Hospital,

Copenhagen, Denmark

2 Institute of Public Health,

University of Copenhagen,

Copenhagen, Denmark

3 Rygestopkonsulenterne Aps

4 Capital Region of Denmark,

Denmark

5 Research Center for Prevention and Health, Denmark

6 Center for Diabetes,

Copenhagen, Denmark

\section{CORRESPONDENCE TO}

Charlotta Pisinger. Center for

Clinical Research and

Prevention, Bispebjerg-

Frederiksberg Hospital,

Hovedvejen, Entrance 5,

Nordre Fasanvej 57, DK-2000

Frederiksberg, Denmark.

E-mail: charlotta.pisinger@

regionh.dk

\section{KEYWORDS}

smoking, smoking cessation, social class, RESPEKT Study, randomized trial

Received: 21 November 2018 Revised: 4 May 2018

Accepted: 22 May 2018

\section{INTRODUCTION}

Smoking is one of the most important determinants of socioeconomic inequality in mortality ${ }^{1}$. Furthermore, spending on tobacco in the poorest households often represents more than a tenth of total household expenditure ${ }^{2}$, leading to high economic stress and less disposable income for food, education and health care. In Denmark, smoking explains the major part of the increase in social inequality in mortality in the last decades ${ }^{3}$.

Despite no difference in intentions to quit or quit attempts, people with low socioeconomic status (SES) are significantly less successful in their quit attempts than those with higher $\mathrm{SES}^{4}$. Some of the reasons for this are strong tobacco addiction, reduced social support for quitting and low self-efficacy ${ }^{5,6}$. 
Unfortunately, it seems that the general smoking cessation interventions in Europe have increased inequalities in smoking ${ }^{7}$, and few studies have tested which interventions are effective among smokers with low $\mathrm{SES}^{8}$. However, there is some evidence that smoking cessation programs targeting persons from lower social classes might have effect ${ }^{5}$, and might reduce inequalities in smoking by increasing the reach to low-SES smokers ${ }^{7}$.

All Danish hospitals have been smoke-free since 2007 and most hospitals have offered their employees assistance to quit but few employees have accepted this offer. Our hypothesis was that we could reduce the smoking prevalence in hospital staff with low socioeconomic status (SES) by involving them in the design, recruitment and conduct of the intervention. Therefore, we designed a randomized intervention to take place in all hospitals in the Capital Region of Denmark (Region H). The study was named RESPEKT (respect in Danish) after the focus group interviews had revealed that many smokers in the target-group expressed being disrespected at the workplace.

This paper presents the design and results of a smoking cessation intervention aimed at and actively involving smoking hospital-employees with low SES. Furthermore, we will describe the unforeseen logistic challenges that led to a change of study design. The longitudinal aspect of the hospital-level assessment was reduced to two cross-sectional surveys but a randomized intervention was performed as planned.

\section{METHODS}

The study was originally designed as a cluster randomized intervention with follow-up at individual level (but not conducted as such, which will be described later) taking place in all hospitals in the Capital Region of Denmark (Region H). One hospital (Bornholm) was excluded as it is situated on a distant island. As some of the hospitals are large and others small we matched the remaining seven hospitals in three groups so the hospitals were more or less comparable in size in the control and intervention groups and then randomly allocated each group to either intervention (the Gentofte, Glostrup, Nordsjællands, and Bispebjerg \&Frederiksberg, hospitals) or control hospitals (the Rigshospitalet, Herlev, and Amager\&Hvidovre, hospitals).
The design of the study was approved by the employees committee and all hospital directors before study commencement. Region $\mathrm{H}$ is running the hospitals and was partner in the research project.

We selected seven occupations as proxy for low SES as they all require less than two years of education and generally have a low income: cleaner, porter, kitchen staff, social and health care assistant, service assistant, technical staff and medical secretary.

In the intervention hospitals smoking employees with low SES were involved in a target-populationtailored intervention (described in detail later).

In the control hospitals everything continued 'as usual'. Smoking employees could contact existing smoking cessation programs at their workplace (typically offering individual counseling after working hours).

The ethical committee of Region $\mathrm{H}$ considered the trial as an improvement of already existing smoking cessation programs (H-3-2013-099) and not as a clinical trial needing ethical approval; therefore, informed consent was not needed. The study was registered in ClinicalTrials.gov (NCT02701530).

\section{Description of the intervention}

First, we performed focus group interviews with the target-group to identify barriers and wishes for smoking cessation. These revealed that even smokers very reluctant to quit at the beginning of the interview had a wish to stopping smoking and would consider participating in a smoking cessation intervention if they received the right offer of assistance. The most important wishes were that a personal smoking cessation counselor would take sufficient time to listen to them, assist with their individual challenges, and encourage and support them - preferably an 'ex-smoker who understands me'. We designed the smoking cessation intervention based on results from the focus group interviews and by involvement of dedicated smokers in the targetgroup. A coordinator identified smoking employees in the target-group who were willing both to stop smoking and to be dedicated 'ambassadors' ( $n=3$ to 5 in every intervention hospital) who would help in designing the intervention program and the invitation letters, help recruiting smoking colleagues, participate in smoking cessation groups, be smoke- 
free role models and motivate colleagues to quit and to stay smoke-free. The ambassadors received approximately US\$1000/€940 each for their work.

\section{The smoking cessation intervention in the intervention hospitals tailored to target-group}

Elements of the peer-designed group-based smoking cessation intervention are shown in Table 1. The flow of the intervention was inspired by the national Gold Standard for group-based smoking cessation ${ }^{9}$, but the topics discussed were chosen by the participants in the groups. All participants received a personal information letter before groups started and a text reminder before each session and were encouraged to support and motivate each other during work time to stay smoke-free. We offered free nicotine products or varenicline by choice, for up to 12 weeks. Nicotine replacement therapy (NRT) was always based on the patch and combined with inhaler or oral spray, and p.r.n. medication was supplied at the second session but was adjusted/changed according to need. The counsellors who ran the smoking cessation (SC-) groups were external professionally trained

Table 1. Elements of the peer-based smoking cessation help tailored to the individual in the RESPEKT study, Capital Region of Denmark, 2015

Small smoking cessation groups (4-6 persons)

Six sessions á 11/2-2 hours (approximately two weeks before quitdate, one week before quit-date, and 3 days, 10 days, 24 days and 6 weeks after quit-date)

All professional counsellors were ex-smokers

Stress management at each session

Good time for recognition of individual challenges and individual encouragement

A text reminder before each session and several encouraging individually tailored text messages during the intervention

Contacted by the counselor if they did not turn up

Lot of humor

Few written materials

Flip-over materials, primarily based on drawings (newly developed)

Very little focus on risk of disease

Nicotine gum was not offered

Free nicotine products or varenicline by choice

Nicotine replacement therapy was based on the patch and combined with inhaler/oral spray p.n.

During worktime, at least partly*

Taking place at workplace

Elements with bold are typically not a part of the Danish Gold Standard smoking cessation intervention. * Two hospitals gave all eight hours off work with pay, while two other hospital managements decided to give four hours only. persons experienced with smoking cessation. The ambassadors from the target-group stopped smoking together with their colleagues and motivated their colleagues to stay smoke-free. The ambassadors had received a very brief education on benefits of smoking cessation before the start of the intervention and they could always get in touch with the professional counsellors and discuss specific cases; they received no formal education on motivation. Self-administered questionnaires were completed during the baseline session. Smoking abstinence was measured at every session as point abstinence and validated by carbon monoxide ( $\mathrm{CO}$ ) levels lower than $8 \mathrm{ppm}$, measured with the Bedfont Micro Smokerlyzer. Six weeks after quit date, at the last group session, all subjects received a questionnaire to evaluate their smoking status and had their CO levels measured. Those who did not attend the last group session were contacted by phone. The SC-groups started in the beginning of January 2015. Six months afterwards, all smokers were contacted by phone by their counselor, and completed a guided telephone survey, and $\mathrm{CO}$ was measured in all persons who reported abstinence from smoking. Two persons did not respond at the 6 months follow-up; one of these had died (excluded), and one had emigrated (considered a smoker with unchanged tobacco consumption, intention-to-treat principle).

\section{Recruitment in the intervention hospitals}

Recruitment was done in three ways: 1) Peer-driven: smoking ambassadors from the target-group were recruiting colleagues at the hospital. Smoking employees know where other smoking employees hide and smoke, and they can better approach their colleagues in a way that will not be considered as judgmental; 2) Written invitations were put in the same envelope as the smoking status survey (in intervention hospitals only). Recruitment materials were designed with guidance from the ambassadors from the target-group and were tailored specifically to each hospital; and 3) Posters were peer-designed showing ambassadors from the target-group in each hospital and their reasons to quit (Fig. 1). They were posted on walls in meeting spaces of employees in the target-group (removed in one hospital). Additionally, one intervention hospital insisted on using the hospital's intranet to inform about the intervention. 
Figure 1. Posters and invitations to take part in the smoking cessation groups. The RESPEKT study, Capital Region of Denmark, 2015

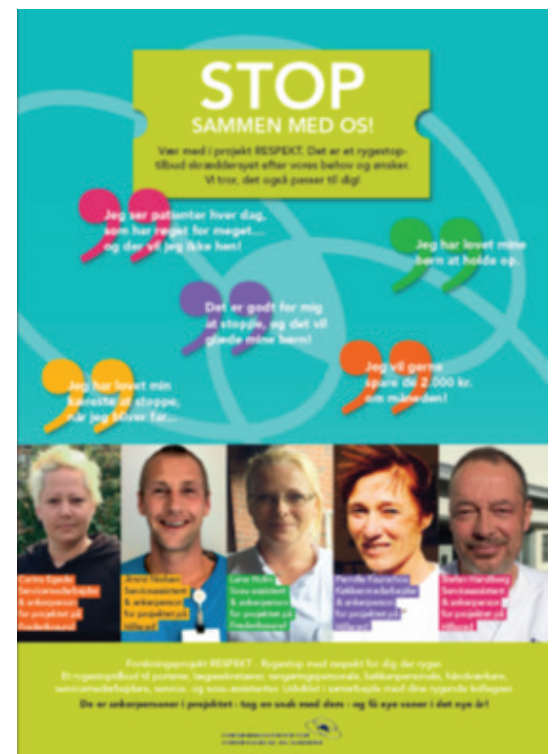

The title of the posters was 'Quit with us'. Photos, names and position of the colleagues (ambassadors) and their reasons to quit filled the entire front. There were statements like: 'I Have promised my children to quit' or 'I have seen too many smoking patients - and I don't want to end there.'

\section{Measuring the effect of the intervention}

In the original design of the study this should have been done by personal letters containing a smoking status survey to all employees in the target-group, independent of randomization status at baseline and one year after the start of the intervention - allowing follow-up of all individuals working in the hospitals at baseline. However, the study could not be conducted as planned. All relevant sectors in the Region $\mathrm{H}$ were contacted many times by both primary investigator and $\mathrm{EH}$ (vice-director in Region $\mathrm{H}$ ) and despite the fact that Region $\mathrm{H}$ was a partner in the study they all directed us to another department/sector or refused to disclose addresses of the employees; neither would the department that had access to addresses send letters to employees in the target-group apparently for legal reasons. They also rejected that the researchers paid for postage and that a student assistant packed the letters at a regional office, so researchers had no access to 'person sensitive' data. Therefore, after months of fruitless negotiation we had to change the design to two cross-sectional surveys at hospital level.

\section{The 'real-life' conduct of the smoking status survey}

We received a list from the Region $\mathrm{H}$ with information on how many employees in the target-group worked

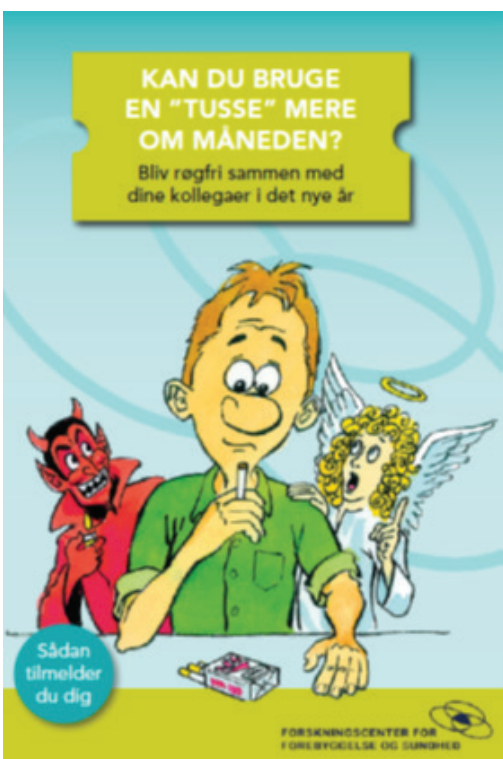

The invitations were designed to be personal, in bright colors, very little text-heavy information stating that this was a research project. A small pamphlet describing the smoking cessation groups had the title: 'Could you use a toad more every month?' (a toad is slang for 1000 Danish Kroner, approximately US $\$ 150 / € 130$, saved by quitting smoking), as the economic aspect of smoking cessation was regarded very motivating. On the back page it was highlighted that there would be no 'pointing fingers' and that the group-based smoking cessation was during work time and together with colleagues.

at each of the 199 hospital departments. We then sent a package of letters to the departments corresponding to the number of employees in the target-group in the department at that time. Letters were sent to all employees in the target-group $(\mathrm{N}=7003)$, independent of randomization status. The letters contained the smoking status survey. Furthermore, employees in the intervention group also received an invitation to take part in the group-based smoking cessation intervention. The head of each department was informed about the research study and asked to deliver the letters to the target-group within a week. As the focus group interviews had revealed that many smokers would not complete a smoking status survey as they were afraid of losing their job, we prioritized anonymity and asked only two questions: 'Do you smoke?' (yes daily; yes occasionally; no but I have smoked previously; no I have never smoked) and 'In which hospital do you work?' (names of intervention hospitals; names of control hospitals). Employees could optionally write their phone number and take part in a prize draw (movie tickets or gift from drug store). Return address was the Research Center. The baseline smoking status survey was performed in the last week of November 2014. As we had no personal information on participants at baseline we could not contact the same employees after one year. Instead we received a new list from the Region $\mathrm{H}$ with 
information on how many employees in the targetgroup worked at each of the hospital departments and repeated the survey in the same way 12 months after startup of the last smoking cessation group, in the first week of February $2016(\mathrm{~N}=7496)$.

\section{Statistical analyses}

Power calculations were performed before the start of the study (as the original randomized design could not be conducted, these can be send on request, see also Appendix 1).

We included only respondents with information on both hospital and smoking status in analyses of one-year effect of the study. We performed simple statistical analyses only, because the results were based on two cross-sectional data-sets, response rates were very low and varied between intervention and control group, and almost doubled at follow-up in the intervention group, and too few participants were recruited in the intervention group. Categorical data were tested by frequencies and chi-squared tests. Outcome was smoking rates in the hospitals at baseline and at one-year follow-up. All data processing was done with the SPSS 22.0 software (SPSS Inc., Chicago, IL, USA). Significance level was set at $5 \%$.

\section{RESULTS}

At baseline 1906 persons responded to the smoking status survey but 30 of them had missing information on hospital and/or smoking status, leaving 666 respondents with information in the intervention group (response rate $17.0 \%$ ) and 1210 in the control group (response rate $39.1 \%$ ). At oneyear follow-up 2340 persons responded but 60 of them had missing information on hospital and/ or smoking status, leaving 1073 respondents with information in the intervention group (response rate $26.7 \%$ ) and 1207 in the control group (response rate $34.7 \%$ ). In the intervention hospitals there was a steep increase (by 38\%) in the response rate from baseline to one-year follow-up but no increase in the control hospitals.

\section{The overall effect of the study}

There was no significant difference in selfreported smoking: 12.8 vs $15.4 \%$ were daily smokers at baseline and 12.4 vs $14.0 \%$ were daily smokers at one-year follow-up, in intervention vs control hospitals, respectively (Table 2 ). In control hospitals there was a significant decrease in self-reported daily smoking prevalence between baseline and follow-up.

Table 2. Smoking status in the RESPEKT study at baseline and at 1-year follow-up (two cross-sectional samples), 1876 respondents at baseline and 2280 at 1-year follow-up

\begin{tabular}{|c|c|c|c|c|c|c|c|c|}
\hline & \multicolumn{2}{|c|}{ Daily smoker } & \multicolumn{2}{|c|}{ Occasional smoker } & \multicolumn{2}{|c|}{ Ex smoker } & \multicolumn{2}{|c|}{ Never smoker } \\
\hline \multicolumn{9}{|c|}{ Intervention hospitals } \\
\hline baseline & 85 & $12.8 \%$ & 32 & $4.8 \%$ & 270 & $40.5 \%$ & 279 & $41.9 \%$ \\
\hline 1-year follow-up & 133 & $12.4 \%$ & 61 & $5.7 \%$ & 398 & $37.1 \%$ & 481 & $44.8 \%$ \\
\hline \multicolumn{9}{|l|}{ Control hospitals } \\
\hline baseline & 186 & $15.4 \%$ & 56 & $4.6 \%$ & 434 & $35.9 \%$ & 534 & $44.1 \%$ \\
\hline 1-year follow-up & 169 & $14.0 \%$ & 69 & $5.7 \%$ & 402 & $33.3 \%$ & 567 & $47.0 \%$ \\
\hline
\end{tabular}

Smoking status at baseline in intervention hospitals compared with control hospitals: $p=0.168$. Smoking status at one-year in intervention hospitals compared with control hospitals: $p=0.262$. Smoking status in intervention hospitals: baseline compared with one-year follow-up: $p=0.413$. Smoking status in control hospitals: baseline compared with one-year follow-up: $p<0.0001$

\section{Effect of the recruitment to SC-groups (intervention hospitals only)}

We sent 3909 letters with invitations to participate in SC-groups to the departments but many employees in the target-group reported that they had never received the letter. Large piles of letters were returned. A total of 119 smokers signed up for the SC-groups but 19 never turned up, leaving
100 smokers who were included; 17 of these were ambassadors from the target-group. We estimate that we recruited almost 10\% (100 of the estimated 938 available smokers, calculation based on Magtengaard Robinson et al.10). Participants reported that being told about the intervention by an ambassador from the target-group or a colleague was the most frequent way of recruitment $(48.2 \%)$. 


\section{Characteristics of smokers in the SC-groups (intervention hospitals only)}

Slightly more women (62\%) than men were included. Mean age was almost 46 years, the median tobacco consumption was 15 cigarettes daily, two out of three smoked within 30 minutes after waking up and $78 \%$

Table 3. Baseline characteristics of the 100 low SES smokers included in the RESPEKT study, Capital Region of Denmark, 2015

\begin{tabular}{|c|c|}
\hline Variable name & $\begin{array}{l}\text { Per cent or } \\
\text { Vean (SD) }\end{array}$ \\
\hline Age (mean) & 45.7 \\
\hline Gender $=$ Female & 62.0 \\
\hline \multicolumn{2}{|l|}{ Position/profession ( $n=99)$} \\
\hline Cleaner & 16.0 \\
\hline Technical staff & 7.0 \\
\hline Porter & 14.0 \\
\hline Social and health care assistant & 10.0 \\
\hline Medical secretary & 17.0 \\
\hline Service assistant & 23.0 \\
\hline Kitchen staff & 13.0 \\
\hline \multicolumn{2}{|l|}{ Motivation to quit before invitation } \\
\hline No intentions to quit & 13.0 \\
\hline Contemplated to quit & 75.0 \\
\hline Planned to quit & 12.0 \\
\hline Previous quit attempts $=$ Yes & 83.0 \\
\hline Age at smoking debut & $16.7( \pm 5.7)$ \\
\hline Mean tobacco consumption & $15.2( \pm 6.5)$ \\
\hline \multicolumn{2}{|l|}{ Use of electronic cigarettes } \\
\hline Daily & 2.0 \\
\hline Occasionally & 13.1 \\
\hline No & 84.8 \\
\hline \multicolumn{2}{|l|}{ Smoking after waking up } \\
\hline Within 5 min & 24.0 \\
\hline $5-30 \mathrm{~min}$ & 43.0 \\
\hline $31-60 \mathrm{~min}$ & 23.0 \\
\hline $60+\min$ & 10.0 \\
\hline Heavy smoking index & $2.6( \pm 1.3)$ \\
\hline \multicolumn{2}{|l|}{ Partner willing to quit? } \\
\hline Lives alone & 38.4 \\
\hline Non-smoking partner & 25.3 \\
\hline Smoking partner, willing to quit now & 18.2 \\
\hline Smoking partner, not willing to quit now & 18.2 \\
\hline \multicolumn{2}{|l|}{ Smoking colleagues/friends/family } \\
\hline None/few & 22.0 \\
\hline Many & 66.0 \\
\hline Most & 12.0 \\
\hline $\begin{array}{l}\text { Confidence in quitting smoking, at first meeting } \\
\text { ( } 0=\text { lowest to } 10=\text { highest) }\end{array}$ & $7.8( \pm 1.9)$ \\
\hline
\end{tabular}

had many/most smokers in their close network/ family (Table 3 ). Noteworthy, $13 \%$ of the smokers stated that they had no intentions to quit before they were invited/nudged by a colleague to participate in the intervention. In $13 \%$ of the cases the counsellor had noted that the participant had problems with abuse (actual or previous) or severe illness (mental or physical; own or in intimate family).

\section{Effect of the SC-groups (intervention hospitals only)}

A total of $89 \%$ participants attended at least four sessions. Six weeks after quit date $79.8 \%$ reported to have quit and were validated as non-smokers (intention-to-treat analyses), most of these had been continuously abstinent. At 6 months follow-up $44.4 \%$ of the 99 participants declared to be smokefree (Table 4 ) - 40.4\% were validated abstinent and $32.4 \%$ were validated as continuously abstinent, not smoking at all from the end of the SC-groups to the follow-up at 6 months (or longer).

Table 4. Abstinence rates 6 months after quit date in the smoking cessation groups in the intervention arm of the RESPEKT study, Capital Region of Denmark, 2015

\begin{tabular}{|c|c|c|}
\hline & $\begin{array}{c}\text { Number } \\
(n=99)\end{array}$ & $\%$ \\
\hline Smoker & 55 & 55.6 \\
\hline Self-reported point abstinence & 44 & 44.4 \\
\hline Validated abstinence ${ }^{*}$ & 40 & 40.4 \\
\hline Continuous abstinence since fixed quit date & 27 & 27.3 \\
\hline Continuous abstinence - at least since last session & 5 & 5.1 \\
\hline Point abstinence - not continuous & 8 & 8.1 \\
\hline
\end{tabular}

${ }^{*}$ Three persons with self-reported abstinence had $\mathrm{CO}>8 \mathrm{ppm}$ and one did not attend c0 measurement.

\section{Other findings in the SC-groups (intervention hospitals only)}

A total of $68 \%$ participants chose nicotine replacement therapy and $27 \%$ chose varenicline. Out of the smokers who had previously received professional assistance for smoking cessation almost all (95\%) stated that this offer was better. Satisfaction with the intervention was very high; mean score 4.9 $( \pm 0.3)$ out of 5 (=highest possible). The counselor's encouragement, the guidance/counseling and the intervention taking place at the workplace, and in 
small groups, were most frequently reported as very important positive factors in the SC-groups. When asked 'What could we have done better?' $83.5 \%$ stated 'nothing'. The most important factors of potential improvement mentioned were 'more sessions' and 'better support from the head of department at work'. Many smokers were not allowed to participate in the SC-groups during working hours, even though they were so promised by their directors.

\section{DISCUSSION}

Even though the hospital directors had approved the study and the Region $\mathrm{H}$ (which runs the hospitals) was partner in the project, we experienced a lack of cooperation once the study started, which seriously affected the study design and the overall results. Follow-up at individual level could not be conducted. The response rates in the smoking status survey and self-reported smoking rates in employees with low SES were very low. Involving the smokers as partners at an early stage of study design facilitated both recruitment and development of the intervention. Despite the high validated long-term abstinence rates in the SC-groups in the intervention hospitals we found no effect of the intervention at hospital-level after one year.

A low response rate, down to $40 \%$, is a general problem in surveys ${ }^{11}$, but the response rates in our survey were even lower. This probably reflects the chaotic design - many employees in the targetgroup never received the letter/survey, the sensitive topic (smoking in a strictly non-smoking workplace), and general low response rates in low-SES groups. The baseline response rate in the smoking status survey was twice as high in the control group as in the intervention group, and we hypothesize that some smokers were negatively affected by the quit smoking offer (invitation to participate in SC-groups in the same letter). Interestingly, the response rates increased by $38 \%$ in the intervention group at the one-year follow-up (no change in the control group), which might reflect a changed positive attitude towards the intervention. Some of the hospitals in the intervention group were challenged by reorganization, which might have contributed to the low response rates.

In total $24 \%$ of citizens with low education in Region $\mathrm{H}$ reported to be daily smokers in $2013^{10}$.
The smoking rates reported in our study were approximately half of this, which most probably reflects major under-reporting; the focus groups had revealed that smokers were afraid of losing their job because of smoking. We found no differences in daily smoking rates in control and intervention hospitals, but due to the very low response rates, the change in study design, and the large difference in response rate from baseline to one-year follow-up in the intervention group, we cannot draw any strong conclusions.

We had expected support from the hospitals whose directors had approved the study but experienced resistance instead. Two hospital directors would not give full-time off work for participation in SC-groups, and even in hospitals where the smokers were promised that they could participate during work time many smokers had to take holiday hours to be able to attend. Also, we experienced a general negative attitude towards smokers in the administrative staff, which is in line with smokers' experiences. The reason might be that most health care professionals are never-smokers with little insight of nicotine dependence. A general stigmatization tendency in the society might also contribute to this ${ }^{12}$.

The Region $\mathrm{H}$ examined their responsibility for the failure of the study and concluded that participation as partner in research studies entails binding responsibilities. In the future, a thorough assessment of whether the Region $\mathrm{H}$ can meet all requirements of partnership in a study will take place before acceptance, so that full commitment can be assured $^{13}$.

A large challenge faced by many smoking cessation programs is recruitment of smokers, and especially reaching those with low SES. A Cochrane review from 2013 on recruitment methods did not identify studies on peer-driven recruitment ${ }^{14}$. To our knowledge, this study is the first to report results of a program using peer-driven recruitment. Half of the smokers were recruited by an ambassador from the target-group or colleague, which partly may be due to problems with delivery of the written invitations. However, the result is promising and should be tested in a randomized design. It is also worth noting that $13 \%$ of the smokers included stated that they had no intentions to quit before they were invited/nudged by a colleague to take part in the intervention. After 
the end of the study the largest of the hospitals in the control group launched a 'traditional' smoking cessation campaign aimed at all smoking employees and offering group-based smoking cessation. Only 7 smokers out of approx. 1600 smokers were recruited.

The Danish national 'Gold Standard' smoking cessation program (primarily group-based) shows impressively high self-reported cessation rates ${ }^{9}$. However, it is well known that selfreports are influenced by social desirability bias, and misclassification rates up to $40 \%$ have been reported $^{15}$. Our study found a misclassification bias of $10 \%$, which is relatively low. When we adjust the self-reported results of the national 'Gold Standard' smoking cessation program for misclassification bias $(10 \%)$, the validated abstinence rate is approximately $20 \%$; in our study in a comparable group of persons not smoking at all from the end of the program to the 6 months follow-up (or longer) we found that $32 \%$ were validated abstinent. Thus, tailoring the SC-groups to smokers' needs seems to increase the validated quit rate by approximately a third.

High smoking prevalence and acceptability of smoking in the network has been identified as some of the most important barriers for smoking cessation - common to all vulnerable groups ${ }^{16}$. In this study, only 2 of 10 smokers had few or no smokers in their family/close network. Quitters experience not only low positive encouragement in their quit attempt but also a negative pressure from the network, e.g. feeling isolated from smoking friends or even teased/bullied as 'health freaks'. The counselor's encouragement was evaluated to be the most important factor for the participants in the intervention. Also, part of the high quit rates achieved in the SC-groups might be explained by support from colleagues ${ }^{17}$. Future interventions should explore strategies to strengthen the non-smoking support to prevent relapse.

\section{Limitations and strengths}

The large size of the study and the involvement of target-groups, in combination with the qualitative research, were strengths. Also, the quit rates in the SC-groups in the intervention arm were validated and there was a follow-up at 6 months.

It is a major weakness that organizational challenges completely changed the original design of the study with follow-up at individual level.
Response rates were low as were recruitment rates; therefore, the study was underpowered to detect potential effects of the intervention. Our choice of target-groups is open to discussion. As a proxy for low SES we chose occupations that require less than two years of education and generally have a low income. We assume that only people with low SES get hired in these low-ranking hospital jobs — which is a weakness. Medical secretaries have a higher education and income than, for example, cleaners and it can be argued that they should not have been included.

\section{CONCLUSIONS}

Unforeseen logistic challenges led to a radical change in study design. Despite high validated long-term abstinence rates in SC-groups in the intervention hospitals, we found no effect of the intervention at hospital-level after one year. However, response rates were very low and so we cannot draw any strong conclusions. Larger involvement of the targetgroup in a stop-smoking program seems feasible and is recommended.

\section{REFERENCES}

1. Kulik MC, Menvielle G, Eikemo TA, Bopp M, Jasilionis D, Kulhanova I et al.: Educational inequalities in three smoking-related causes of death in 18 European populations. Nicotine Tobacco Research 2014 , 16(5):507-518. doi:10.1093/ntr/ntt175

2. Thomson GW, Wilson NA, O'Dea D, Reid PJ, HowdenChapman P: Tobacco spending and children in low income households. Tobacco Control 2002, 11(4):372375. doi:10.1136/tc.11.4.372

3. Koch MB, Diderichsen F, Gronbaek M, Juel K: What is the association of smoking and alcohol use with the increase in social inequality in mortality in Denmark? A nationwide register-based study. BMJ Open 2015, 5(5):e006588. doi:10.1136/bmjopen-2014-006588

4. Kotz D, West R: Explaining the social gradient in smoking cessation: it's not in the trying, but in the succeeding. TobControl 2009, 18(1):43-46.

5. Hiscock R, Bauld L, Amos A, Fidler JA, Munafo M: Socioeconomic status and smoking: a review. Annals of the New York Academy of Sciences 2012, 1248:107-123. doi:10.1111/j.1749-6632.2011.06202.x

6. Siahpush M, McNeill A, Borland R, Fong GT: Socioeconomic variations in nicotine dependence, selfefficacy, and intention to quit across four countries: findings from the International Tobacco Control (ITC) Four Country Survey. Tobacco Control 2006, 15 Suppl 3:iii71-iii75. doi:10.1136/tc.2004.008763 
7. Brown T, Platt S, Amos A: Equity impact of European individual-level smoking cessation interventions to reduce smoking in adults: a systematic review. European Journal of Public Health 2014, 24(4):551-556. doi:10.1093/eurpub/cku065.

8. Courtney RJ, Naicker S, Shakeshaft A, Clare P, Martire KA, Mattick RP: Smoking Cessation among Low-Socioeconomic Status and Disadvantaged Population Groups: A Systematic Review of Research Output. International Journal of Environmental Research and Public Health 2015, 12(6):6403-6422. doi:10.3390/ijerph1206064039.

9. Neumann T, Rasmussen M, Ghith N, Heitmann BL, Tonnesen H: The Gold Standard Programme: smoking cessation interventions for disadvantaged smokers are effective in a real-life setting. Tobacco Control 2013, 22(6):e9. doi:10.1136/tobaccocontrol-2011-050194

10. Magtengaard Robinson K, Lykke M, Helbech Hansen B, Jeppesen M, Prip Buhelt L, Juel C, Glümer C: Sundhedsprofil for region og kommuner 2013. In. Region Hovedstaden, Center for Sundhed: Forskningscenter for Forebyggelse og Sundhed, 2014.

11. Mindell JS, Giampaoli S, Goesswald A, Kamtsiuris P, Mann C, Mannisto S, Morgan K, Shelton NJ, Verschuren WM, Tolonen H: Sample selection, recruitment and participation rates in health examination surveys in Europe-experience from seven national surveys. BMCMed ResMethodol 2015, 15:78.

12. Castaldelli-Maia JM, Ventriglio A, Bhugra D: Tobacco smoking: From 'glamour' to 'stigma'. A comprehensive review. Psychiatry ClinNeurosci 2016, 70(1):24-33. Castaldelli-Maia JM, Ventriglio A, Bhugra D: Tobacco smoking: From 'glamour' to 'stigma'. A comprehensive review. Psychiatry Psychiatry and Clinical Neurosciences 2016, 70(1):24-33. doi:10.1111/pcn.12365

13. Vicedirektor Center for Sundhed Else H, Personalepolitisk chef for Enhed for Organisation og Ledelse Svend Skovmand E: Respektstudiet. In.; 2016.

14. Marcano Belisario JS, Bruggeling MN, Gunn LH, Brusamento S, Car J: Interventions for recruiting smokers into cessation programmes. Cochrane Database Syst Rev 2012, 12:CD009187. doi: 10.1002/14651858.cd009187.pub2

15. Jamrozik K, Vessey M, Fowler G, Wald N, Parker G, van Vunakis $H$ : Controlled trial of three different antismoking interventions in general practice. $\mathrm{Br}$ Med J (Clin Res Ed) 1984, 288(6429):1499-1503. doi: 10.1136/bmj.288.6429.1499

16. Twyman L, Bonevski B, Paul C, Bryant J: Perceived barriers to smoking cessation in selected vulnerable groups: a systematic review of the qualitative and quantitative literature. BMJ Open 2014, 4(12):e006414. doi:10.1136/bmjopen-2014-006414

17. Cahill K, Lancaster T: Workplace interventions for smoking cessation. Cochrane Database Syst Rev 2014,
2:CD003440. doi: 10.1002/14651858.cd003440.pub3
ACKNOWLEDGEMENTS

We thank all persons participating in the study, especially the ambassadors from the target-groups and the smoking cessation counsellors.

CONFLICTS OF INTEREST Dr Pisinger and Dr Bjerre Koch reports grants from Tryg Foundation, during the conduct of the study. The rest of the authors also have completed and submitted an ICMJE form for disclosure of potential conflicts of interest. The authors declare that they have no competing interests, financial or otherwise, related to the current work.

FUNDING

This work was supported by the Danish Tryg Foundation (TrygFonden), ID 102861.

PROVENANCE AND PEER REVIEW

Not commissioned; externally peer reviewed. 